\title{
Observation of Viruses, Bacteria, and Fungi in Clinical Skin Samples under Transmission Electron Microscopy
}

\author{
Yuping Ran, Wengying Hu, Kaiwen Zhuang, Mao Lu, \\ Jinghong Huang, Fengni Xu, Xiaoxi Xu, Xia Hua, Jebina Lama, \\ Xin Ran, Yalin Dai and Song Lei
}

Additional information is available at the end of the chapter

http://dx.doi.org/10.5772/60957

\begin{abstract}
The highlight of this chapter is the description of the clinical manifestation and its pathogen and the host tissue damage observed under the transmission electron microscopy, which helps the clinician understand the pathogen's ultrastructure, the change of host sub-cell structure, and helps the laboratory workers understand the pathogen-induced human skin lesions' clinical characteristics, to establish a two-way learning exchange database with vivid images.
\end{abstract}

Keywords: Viruses, bacteria, fungi, clinical skin samples, TEM

\section{Introduction}

In the Dermatovenereology department, skin infections by bacteria, viruses, and fungi are very common in routine clinical practice. Discrimination and identification of these pathogens is a huge challenge and very important for patient's disease diagnosis and treatment. Transmission electron microscopy (TEM) is a very strong tool for detection and observation of the pathogen from the clinical samples that help us obtain the direct proof of presence of the pathogen inside the skin samples of the lesion. Based on the detailed morphologic image, we can recognize the ultrastructures of the pathogen and understand the pathogenesis of the infectious skin diseases. During recent years, we collected several pathogenic microorganisms' photographs which were taken by TEM; these pathogens included viruses (Herpes simplex virus, Varicella- 
zoster virus, Molluscum contagiosum virus), bacteria (Mycobacterium leprae), and fungi (Trichophyton violaceum, T. tonsurans, T. mentagrophytes, Trichosporon inkin, Penicillium marneffei). The diagnosis and clinical manifestation, the source of sample, and the image of the pathogen are summarized in Table 1.

\begin{tabular}{|c|c|c|c|}
\hline Diagnosis & Clinical manifestation & Sample & Image of pathogen by TEM \\
\hline Herpes simplex & $\begin{array}{l}\text { Painful grouped vesicles, pustular, ulcer, crust } \\
\text { usually on the skin; oral, labial, and ocular mucosa. }\end{array}$ & Blister wall & Virus (Herpes simplex virus) \\
\hline Herpes zoster & $\begin{array}{l}\text { Erythema, papule, vesicles, or bullous distributed } \\
\text { unilaterally within the peripheral nerves. }\end{array}$ & Blister wall & Virus (Varicella-zoster virus) \\
\hline $\begin{array}{l}\text { Molluscum } \\
\text { contagiosum }\end{array}$ & $\begin{array}{l}\text { Dome-shaped papules of 3-5 } \mathrm{mm} \text { in diameter with a } \\
\text { waxy appearance on the surface and a white } \\
\text { curd-like core. }\end{array}$ & Papule & $\begin{array}{l}\text { Virus (Molluscum contagiosum } \\
\text { virus) }\end{array}$ \\
\hline Leprosy & $\begin{array}{l}\text { Erythematous patches or papules, alopecia, leproma; } \\
\text { thickening of peripheral nerves, neurological } \\
\text { disorders. }\end{array}$ & Skin tissue & $\begin{array}{l}\text { Bacteria (Mycobacterium } \\
\text { leprae) }\end{array}$ \\
\hline Tinea capitis & $\begin{array}{l}\text { Erythema, papules, scales, and pustule on the scalp; } \\
\text { broken hair, and hair loss. }\end{array}$ & $\begin{array}{l}\text { Infected } \\
\text { hair }\end{array}$ & $\begin{array}{l}\text { Fungus (Trichophyton } \\
\text { violaceum; T. tonsurans) }\end{array}$ \\
\hline $\begin{array}{l}\text { Trichomycosis } \\
\text { nodularis }\end{array}$ & $\begin{array}{l}\text { White or brown nodules surround the pubic or } \\
\text { armpit hair shaft, the nodule is soft. }\end{array}$ & $\begin{array}{l}\text { Infected } \\
\text { hair }\end{array}$ & Fungus (Trichosporon inkin) \\
\hline $\begin{array}{l}\text { Penicilliosis } \\
\text { marneffei }\end{array}$ & $\begin{array}{l}\text { Fever, anima, superficial lymphadenopathy, } \\
\text { hepatosplenomegaly, polymorphic skin lesions on } \\
\text { face, torso, and extremities. }\end{array}$ & Skin tissue & Fungus (Penicillium marneffei) \\
\hline
\end{tabular}

Table 1. The summary of diagnosis and clinical manifestation, kinds of sample, and the image of the pathogen observed by TEM

\section{Methods}

All samples for TEM were taken from clinical patients. These samples include blister wall, papule, infected hair, scales, and biopsy tissue. The samples were double-fixed in $2 \%$ glutaraldehyde and $2 \%$ osmium tetroxide for $3 \mathrm{~h}$ at the room temperature, dehydrated in series of grade ethanol solutions and propylene oxide, then embedded in resin. Ultrathin longitudinal sections of infected hair were cut with an ultramicrotome and a diamond knife. Observation was carried out by TEM (Hitachi H-7650 microscope), which was operated at $120 \mathrm{kV}$ and equipped with a LaB6 source.

\section{Results}

\subsection{Herpes simplex}

Herpes simplex is a viral dermatosis caused by Herpes simplex virus (HSV) infection, and characterized by painful grouped vesicles on the skin or mucous membranes, mainly on the 
mucocutaneous junction. It is self-limiting, but tends to recur frequently. HSV, a member of alpha subfamily of the Human herpes virus, is a double-stranded DNA virus that affects humans only. It is sub-classified as type 1 and type 2 according to the differences in its transmission modes and clinical features. One of the important biological properties of alpha herpes virus is its ability to establish latent infections in neurons. When HSV encounters broken skin or a mucosal surface, viral replication occurs first at the point of entry, initiating a primary infection. The virus then invades in local nerve endings, where, latency is established. In the latent stage, the virus resides in infected ganglia in a nonreplicating state and persists for the lifetime of the host. Several provocative stimuli can reactivate virus from the latent stage: transient immunosuppressive states of the host induced by fever, gastrointestinal disturbance, menstruation, or stress. During the reactivation, the latent virus returns to the peripheral sites of the skin or mucous membranes and replicates to produce an outbreak of recurrent herpes simplex. The aim of herpes simplex treatment is to shorten the current outbreak, to prevent secondary bacterial infections, and to avoid or postpone recurrence [1,2]. The frequency and severity of recurrent outbreaks vary greatly among people. Some individuals' outbreaks can be quite debilitating, with large, painful lesions persisting for several weeks, while others may experience only minor itching or burning for a few days. Immunity to the virus is built over time. Most infected individuals experience fewer outbreaks and outbreak symptoms often become less severe. After several years, some people become perpetually asymptomatic and no longer experience outbreaks, though they may still be contagious to others. Immunocompromised individuals may experience longer, more frequent and severer episodes. Antiviral medication has been proven to shorten the frequency and duration of outbreaks [3].

We describe a case of herpes simplex. A seven-year-old girl presented at our clinic with the complaint of painful blisters and erosions on right eyelid and lower left eyelid for five days (Fig. 1a). Sample was taken from the blister's wall for TEM observation. A large number of electron empty, particle, and dense cores were discovered, which were the characteristics of Herpes simplex virus (Fig. 1b). The patient was diagnosed with bilateral eyelid herpes simplex and was cured by anti-virus therapy.

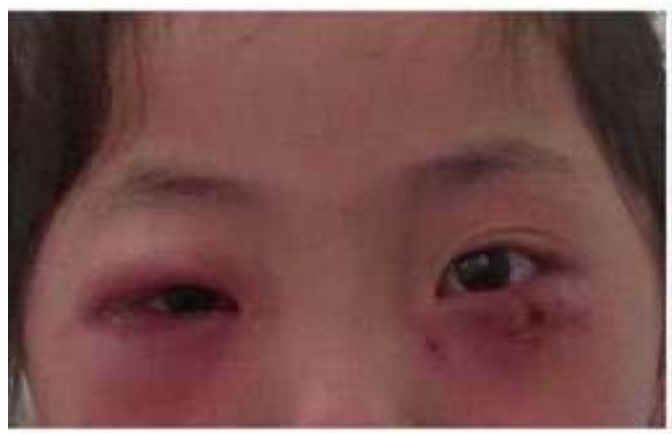

(a)

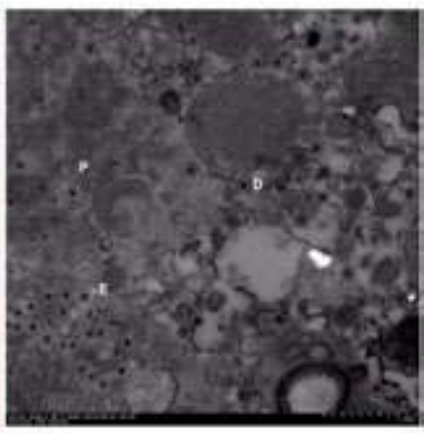

(b)

Figure 1. a. A 7-year-old girl presented at our clinic with the complainant of painful blisters and erosions on right eyelid and lower left eyelid for five days. The patient's eyelids were swollen significantly, white-like substance was observed on the right corner, the left lower eyelid was covered with brown crust. b. TEM revealed that the cellus membrane dissolved the mitochondria and the nucleus was swollen. 


\subsection{Herpes zoster}

Herpes zoster is a viral disease characterized by a painful skin rash with erythema, papule, vesicles, or bullous in a limited area (often in a stripe) on one side of the body (left or right) [4]. The initial infection with Varicella-zoster virus (VZV) causes the chickenpox which generally occurs in children and young adults. The virus is then transported by retrograde axonal flow to the dorsal root ganglia, where, after further replication, latency is established. If the VZV is not eliminated from the body, it can go on to cause herpes zoster. Typical clinical manifestations and laboratory tests (VZV-specific IgM antibody, VZV DNA, electron microscopy for virus particles, etc.) can diagnose herpes zoster. Antiviral agents, analgesics, or even steroids can be used for the treatment. Rash and acute neuritis are the most common clinical manifestations of herpes zoster. Herpes zoster usually starts as erythema and papule, which change to vesicles or bullous quickly and may occasionally change to pustular or hemorrhagic in few days. Pain is the most common symptom which feels like burning, throbbing, or stabbing. The sensation can be prodromal or simultaneous. When the patient gets facial paralysis, ear pain, and vesicles in the auditory canal and auricle, it is called Ramsay Hunt syndrome. Postherpetic neuralgia is the most commonly found sequela. Severe or recurrent zoster usually happens in immunocompromised host such as patients with HIV infections or AIDS. Timely treatment can relieve symptoms and shorten the course of disease.

We describe a case of herpes zoster. The patient was a 36-year-old man who had suffered for painful vesicles for four days (Fig. 2a) on his left back and chest. The diagnosis of herpes zoster was established by the typical clinical manifestations and the numerous viruses under TEM (Fig. 2b). He was cured after taking brivudine tablet $125 \mathrm{mg}$ per day for a week.

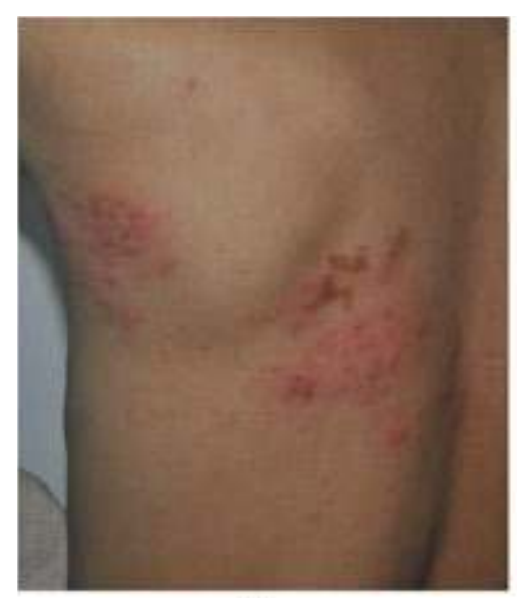

(a)

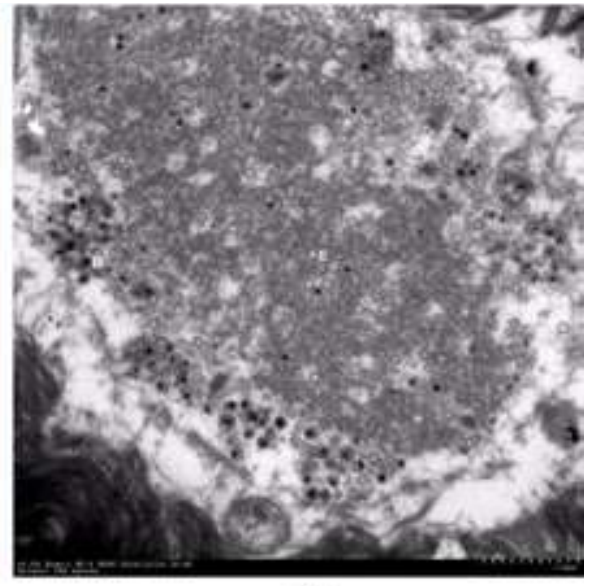

(b)

Figure 2. a. The patient was a 36-year-old man who had suffered from painful vesicles for four days on his left back and chest. b. TEM revealed that the cellus membrane dissolved. A large number of electron empty (E) and dense (D) of VZV nucleocapsids in the nucleus and these nucleocapsids were generally $100 \mathrm{~nm}$ in diameter. 


\subsection{Molluscum contagiosum}

Molluscum contagiosum (MC) is a viral infection of the skin or occasionally of the mucous membranes, which is caused by Molluscum contagiosum virus (MCV, a double-stranded DNA poxvirus) [5]. Skin lesions classically present as flesh-colored papules of 3 to $5 \mathrm{~mm}$ in diameter with central umbilication and a white curd-like core [6]. It has a higher incidence in children, sexually active adults, and those who are immunodeficient, and the infection is most common in children aged one to ten years [7]. Various regions of the body are usually affected, plantar localization of MC is uncommon and can cause pain on walking. Secondary bacterial infection can occur, particularly if patients are scratching their lesions. Inflammatory reaction to $\mathrm{MCV}$ is called molluscum dermatitis, inflamed MC lesions, which can lead to reactions like GianottiCrosti syndrome [8]. Typical clinical appearance or laboratory tests (histology, electron microscopy, etc.) can make diagnosis definite. A vaccine for this infection is not available. Removal by curettage then topical use antiviral is widely used and effective.

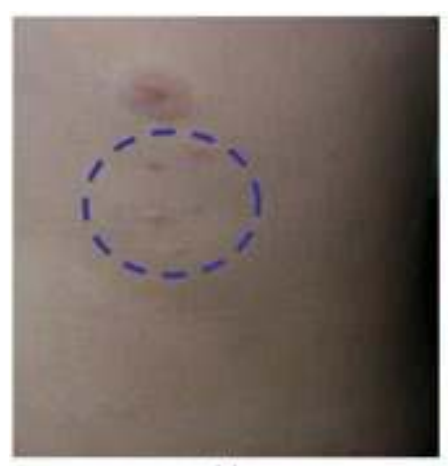

(a)

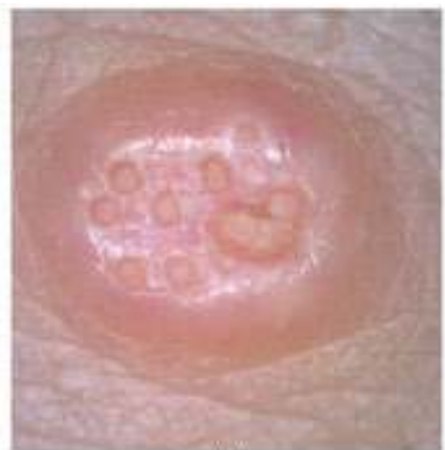

(b)

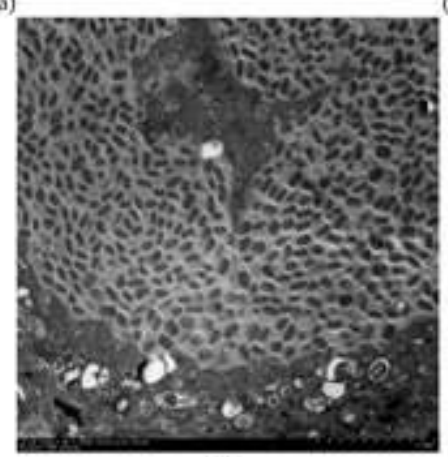

(c)

Figure 3. a. A 10-year-old boy presented at our clinic who wanted to know what the cuticolor papule on his chest was, which appeared one year before. Also, he did not feel pruritus or pain or anything else about the papule. b. Under the dermoscopy, the hemispheric papule was shiny and waxy, with several molluscum bodies on the top, just like cheese. c. TEM revealed that the cytoplasm was full of MCV nucleocapsids which were of the same size as 200 to $300 \mathrm{~nm}$. 
We describe a case of MC on chest. The patient was a 10-year-old boy who presented at our clinic to know what the cuticular papule on his chest was, which appeared one year before, also he did not feel pruritus or pain or anything else about the papule (Fig. 3a). Under the dermoscopy, the hemispheric papule was shiny and waxy, with several molluscum bodies on the top, just like cheese (Fig. 3b). In the TEM, the cytoplasm was full of nucleocapsids which were of the size 200 to $300 \mathrm{~nm}$ (Fig. 3c). The boy was cured after scraping the skin lesions and topically using $2 \%$ tincture of iodine.

\subsection{Genital molluscum contagiosum}

We describe a case of genital MC. The patient was a woman aged 31 years who had suffered for light pruritus papules on her vulva for nearly a week (Fig. 4a), the proximal skin of her left thigh suffered from tinea cruris. The diagnosis of genital MC was established by clinical manifestations and the numerous MCV under TEM (Fig. 4b). She was cured after removal by curettage per week and topical imiquimod cream three times a week for two weeks.

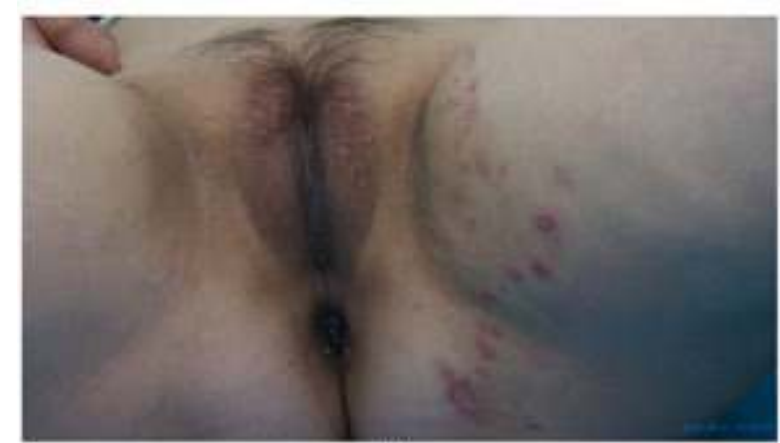

(a)

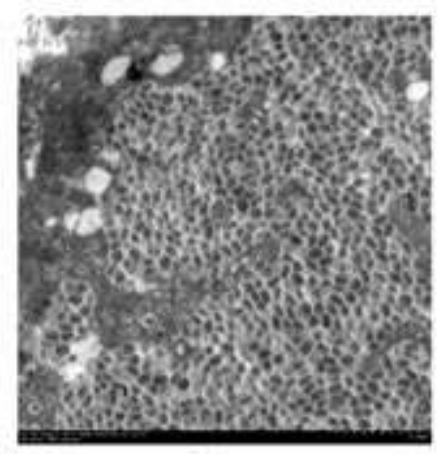

(b)

Figure 4. a. A female patient of 31 years old presented at our clinic whose vulva and pubic area had developed pruritus papules for nearly a week. Physical examination revealed the papules of 3 to $5 \mathrm{~mm}$ in diameter with a waxy appearance on the surface, some of which had atypical central umbilication and a white curd-like core. The proximal skin of her left thigh suffered from tinea cruris with target-like erythema and scales. b. TEM of the central waxy core revealed the strands of cytoplasmic material separating pockets of mature MCV which were generally $300 \mathrm{~nm}$ in length and $100 \mathrm{~nm}$ in height.

\subsection{Leprosy}

Leprosy, formerly known as Hansen's disease, is a chronic infection caused by Mycobacterium leprae, and mainly involves the skin and peripheral nerves. It is classified as tuberculoid (TT), borderline tuberculoid (BT), mid-borderline or borderline (BB), borderline lepromatous (BL), and lepromatous (LL), according to the cellular immunity against M. leprae [9]. In TT, there are few erythemas, a small number of papules, and slightly reduced sensory perception. In LL, M. leprae proliferate in the entire body to form lepromas, multidrug therapy including dapsone is the main treatment. The mechanism of infection has not been fully clarified. Because 
the pathogenicity of $M$. leprae is extremely low, the natural immune response normally eliminates the infection. The incubation period is about 3 to 5 years. This makes the early diagnosis of the disease difficult. Leprosy occurs worldwide, with half of the world's (approximately 12 million) cases occurring in Asia and Africa. Skin lesions of leprosy can be single or multiple, usually hypopigmented, although occasionally reddish or copper-colored. The lesions may be macules (flat), papules (raised), or nodular. Thickened nerves are associated with leprosy and can be accompanied by loss of sensation or muscle weakness. People with leprosy often encounter discrimination. Depending on the level of disfigurement, a patient with leprosy receives varying stigma and ostracism [10].

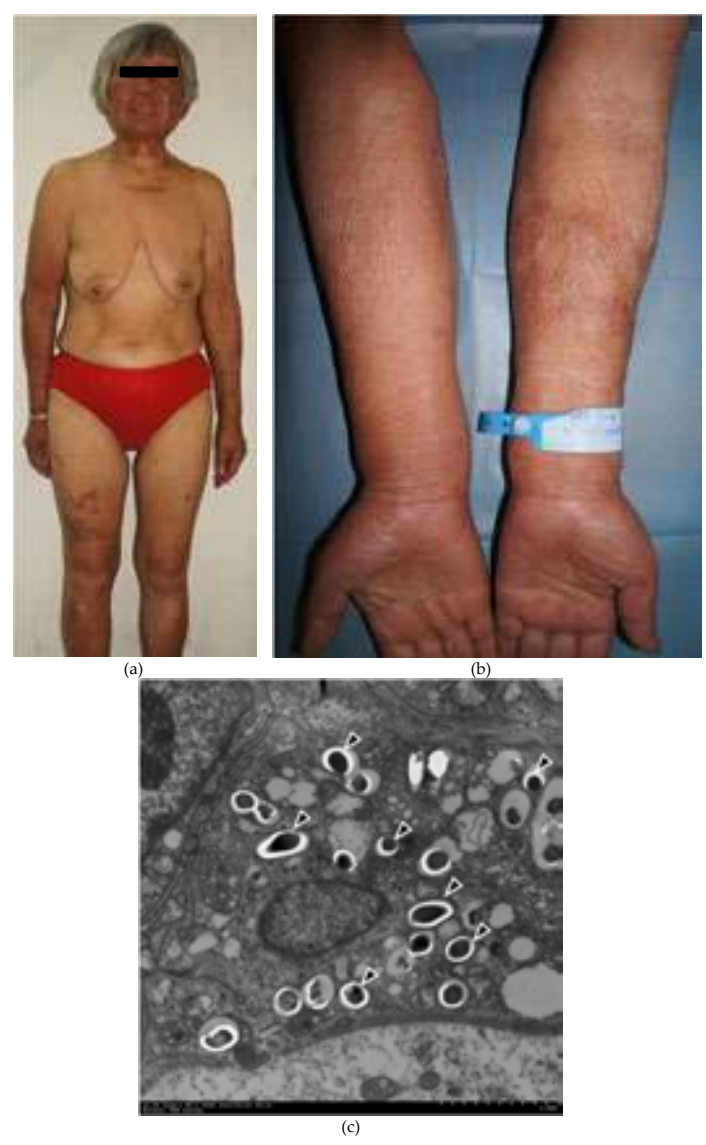

Figure 5. a. A 64-year-old woman consulted of the lesion developed over her whole body for 2 years. Physical examination showed the facial visible invasive dark red plaques, eyebrows lose off. Dark red macules and subcutaneous nodules could be seen scattered on her trunk, limbs. b. Close look of dark red macules and subcutaneous nodules on the arms of the patient. c. A part of biopsy tissue from left waist lesion was processed for TEM studies. The arrows point to bacteria of Mycobacterium leprae inside the Langerhans cell. The length of the bacilli was about 2000 to $6000 \mathrm{~nm}$, the width was 200 to $600 \mathrm{~nm}$. 
We describe a case of leprosy. A 64-year-old woman presented at our clinic with the complaint of dark red macules and subcutaneous nodule on whole body for 2 years (Figs. 5a, 5b). A part of biopsy tissue from her arm was processed for TEM studies (Fig. 5c). The patient was diagnosed with leprosy and cured with anti-leprosy therapy.

\subsection{Tinea capitis}

Tinea capitis is a common superficial fungal infection of scalp hair follicles, hair shaft, and, surrounding skin. It often affects children while rarely adults. Its pathogens are dermatophytes, usually species of the genera Microsporum and Trichophyton, such as Microsporum canis, Trichophyton tonsurans, T. violaceum, and T. mentagraphytes [11]. The clinical manifestation of tinea capitis is highly variable, depending on the causative organism, type of hair invasion, and degree of host inflammatory response. Common features are patchy hair loss with varying degrees of scaling and erythema. In kerion, the lesions were characterized by pustules in different sizes, which can lead to scarring alopecia. However, the clinical signs may be subtle and diagnosis can be challenging. A number of clinical patterns exist [12]. The accurate diagnosis of tinea capitis usually depends on the laboratory investigation, mainly including direct microscopic examination (DME) with $10 \%$ to $30 \%$ potassium hydroxide, and fungal culture. It can confirm the diagnosis to detect or isolate the causal organism either by these two methods. Tinea capitis always requires systemic antifungal treatment. Topical treatment is only used as adjuvant therapy to systemic antifungal agents, as topical antifungal agents do not penetrate the hair follicle. Recommended drug in systemic treatment include itraconazole, terbinafine, and griseofulvin.

We describe two cases of tinea capitis due to T. violaceum [13] and T. tonsurans. Both species of dermatophytes invade only the inner area of the hair shaft, called "endothrix." The patient suffering from tinea capitis caused by $T$. violaceum was a 9-year-old boy, weighing $25 \mathrm{~kg}$ presented to our clinic because of multiple, slightly itching, and patchy areas of gray scales on the scalp associated with hair loss (Fig. 6a). The diagnosis of tinea capitis caused by $T$. violaceum was established by DME, culture, and slide-culture. Under the TEM, the transected section of infected hairs revealed abundant fungus within the hair shaft (Figs. 6b, 6c). The boy was cured after receiving 4 weeks of systemic treatment with itraconazole $125 \mathrm{mg}$ per day and topical treatment with $1 \%$ naftifine- $0.25 \%$ ketaconazole cream once a day. Another patient was a 2-year-old girl, with scalp erythema, scaling for 2 months, and previously healthy. Dermatologic examination was as follows: several erythema, scales were visible on scalp, did not see obvious papules, pustule, and hair loss (Fig. 7a). Laboratory examination results were as follows: Hyphae were seen by DME with $10 \% \mathrm{KOH}$ of the scales from hair. Fungal culture of hair revealed white villiform colonies which were identified as T. tonsurans by ITS1/4-PCR, sequence-based molecular validation. When examined through TEM, it showed many fungal spores inside the infected hair shaft (Fig. 7b). The patient was diagnosed with tinea capitis caused by $T$. tonsurans. She was prescribed itraconazole capsule $50 \mathrm{mg}$ per day for 4 weeks and her clinical condition was improved. 


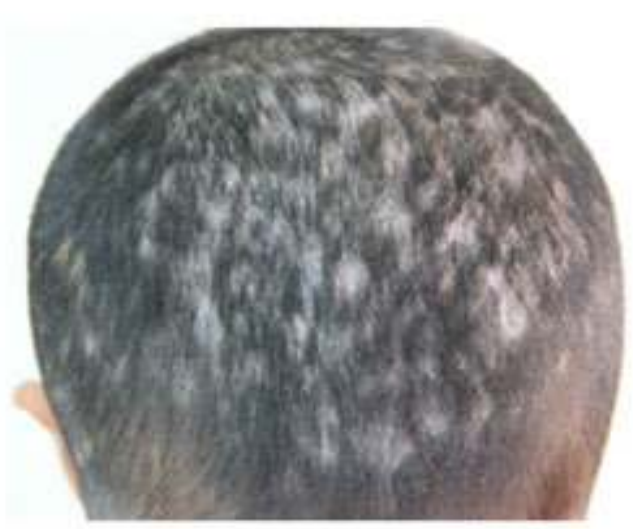

(a)

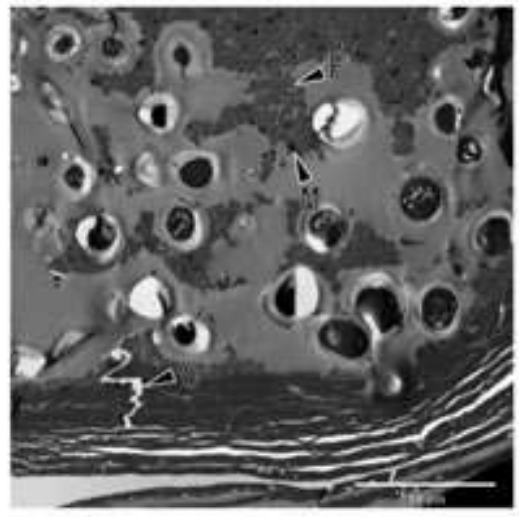

(b)

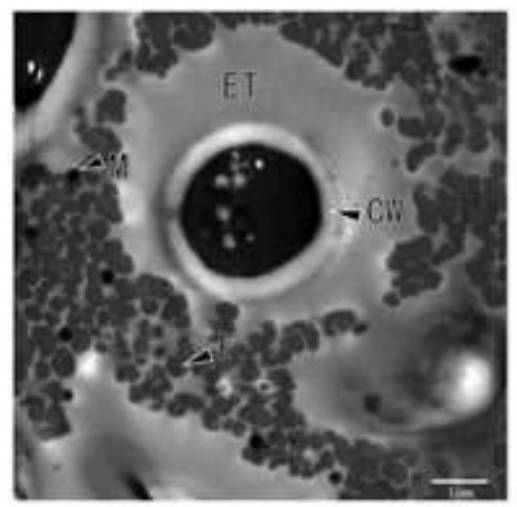

(c)

Figure 6. a. A patient with tinea capitis caused by T. violaceum is a 9-year-old boy, weighing $25 \mathrm{~kg}$ presented to our clinic because of multiple, slightly itching, and patchy areas of gray scales on the scalp associated with hair loss. $b$. Cross-section of the affected hairs showing abundant arthroconidias and residual keratinized fibrils $(\mathrm{F})$ in which menalosomes (M) scattered, a crack (C) in the separated cuticle layer. c. High magnification of cross-section of cortex of the affected hairs: the endothrix arthrospore with cell wall (CW), electron-transparent zone (ET), residual hair-macrofibril bundles (F), and the melanin pigment particles (M).

\subsection{Trichomycosis nodularis}

Trichomycosis nodularis, so called as piedra, is a fungal infection of the hair shaft, which has been subclassified into white piedra (WP) and black piedra, caused by Piedraia hortae. WP is often caused by the Trichosporon genus including T. cutaneum, T. asahii, T. mucoides, T. ovoides, T. asteroides, and T. inkin, all of which belong to the class of Basidiomycetes [14] and can be the 


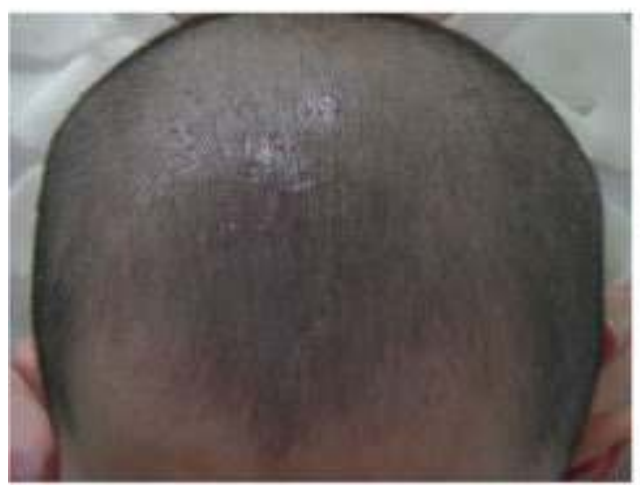

(a)

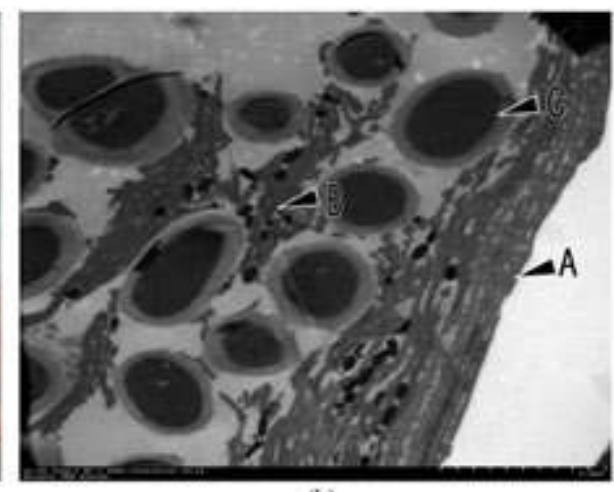

(b)

Figure 7. a. A 2-year-old girl presented with erythema and scaling on her scalp for 2 months. Several erythema, scales were visible on scalp, did not see obvious papules, pustule, and hair loss. b. Cross-section of the infected hair. It shows the cortex of the affected hairs (A), the damaged fragment of hair medulla (B), and endothrix fungal spores (C).

resources of systematic infection especially to immunodeficient patients. This condition predominantly occurs in tropical and subtropical regions. Humidity, hyperhidrosis, and poor personal hygiene are important predisposing factors. The clinical appearance of WP is characterized by concretions or soft, whitish nodules on hair shaft. WP can be found in a variety of hairy areas including the scalp, beard, moustache, genitals, axilla, and even eyebrows and eyelashes [15]. The scalp lesions of white piedra are usually asymptomatic and can be misdiagnosed as pediculosis. DEM of infected hair and culture can confirm the diagnosis. In treatment, shaving off the infected hairs and topical use of antifungal agents are the paramount regime [16].

We describe a case of white piedra due to T. inkin. The patient was a 30-year-old African man. The dermoscopy reveals discrete yellowish concretions randomly distributed on the curly shafts of his pubic hairs (Fig. 8a). The diagnosis of WP caused by T. inkin was established by DME, culture, and molecular study. Under the TEM, the longitudinal section of the infected pubic hair revealed that the yellowish nodule was full of many spores. Spores were closely attached with each other with intercellular materials of cell wall, lower electronic dense vacuole, and nuclear inside the spores were noted (Fig. 8b). The patient was cured after complete shaving, clearance of pubic hairs, oral itraconazole, and topical treatment with $2 \%$ ketaconazole shampoo once a day.

\subsection{Penicilliosis marneffei}

Penicillium marneffei is the only dimorphic pathogenic species in the genus, making penicilliosis marneffei mainly epidemic in the Southeast Asia, which includes southern China. The patients 


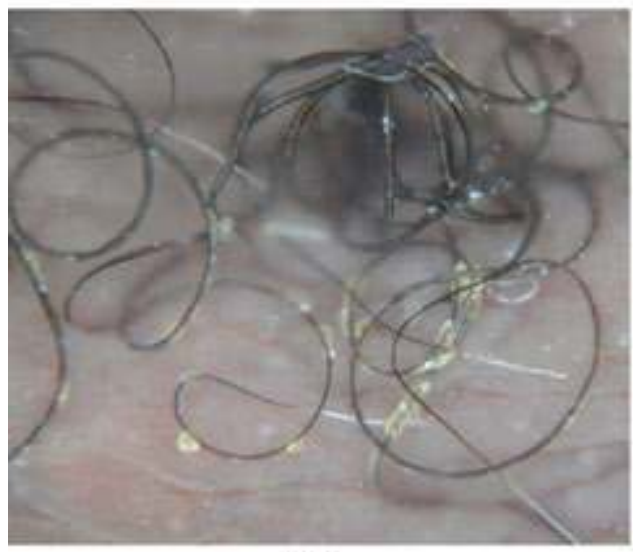

(a)

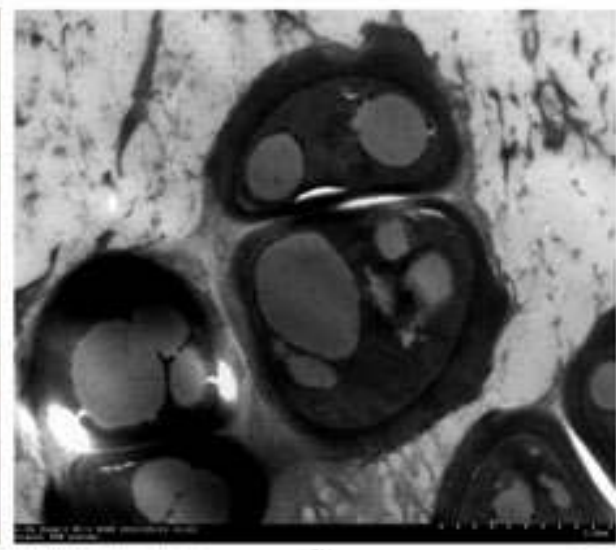

(b)

Figure 8. a. The patient is a 30-year-old African man, having uneasiness in his pubic hairs, having some dirty materials attached to them. Under the dermoscopy, some yellowish nodules attached to the shaft of pubic hairs. b. TEM revealed the internal structure of those attachments, which were full of many spores. Closely attached with each other with intercellular materials of cell wall, lower electronic dense vacuole, and nuclear inside the spores were noted.

infected with HIV/AIDS are the most common susceptible population of $P$. marneffei infection, which is the marker of screening the HIV/AIDS patients. P. marneffei appear at $25^{\circ} \mathrm{C}$ for mycelial form, and at $37^{\circ} \mathrm{C}$ for yeast form. The former in the culture medium can form the wine-like pigment, the latter does not. Only yeast form can be seen inside the infectious tissue. $P$. marneffei violate mononuclear phagocyte system and the main clinical manifestations were fever, anima, superficial lymphadenopathy, and hepatosplenomegaly. P. marneffei in host macrophages is always mostly round or oval shape, about $3 \mu \mathrm{m}$ in diameter. The yeast cells of $P$. marneffe $i$ with septate formed during binary fission. Sometimes we can find macrophages phagocytosing yeast cells [17]. A few lymphocytes or other inflammatory cells are formed in the macrophages. When macrophages were destroyed, yeast was temporarily free from cells [18]. Drug sensitivity test in vitro showed that P. marneffei is highly sensitive to itraconazole and ketoconazole. The prognosis of patients with P. marneffei infection is poor. Although the use of antifungal drugs can improve the prognosis, the mortality rate has reached $20 \%$. Research shows that, although the effect is good, there are still about $50 \%$ of patients in the initial treatment of relapse six months after remission.

We describe a case of penicillinosis marneffei. The patient was a 39-year-old man who presented at our clinic with the chief complaint of swelling in left side of the cheek for four months duration (Fig. 9a). Under the TEM, six oval-shaped phagocytosed yeast cells, within distended histocytes, were observed (Fig. 9b). Among them, the highlight for differentiating P. marneffei yeast from other organisms was the characteristic septum of cell (Fig. 9c). The patient was cured with taking itraconazole for 3 months. 


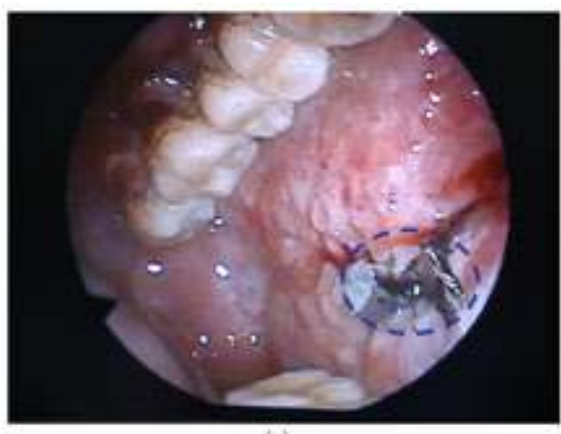

(a)

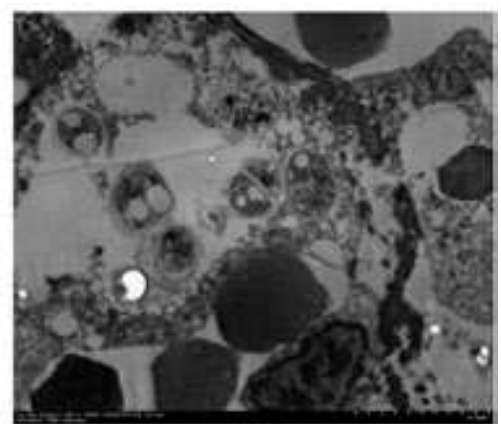

(b)

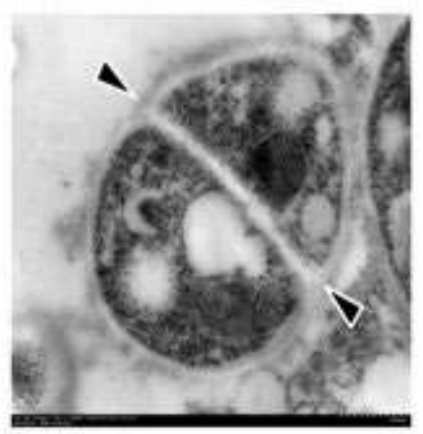

(c)

Figure 9. a. A 39-year-old man who presented at our clinic with the chief complaint of swelling in left side of the cheek for 4 months duration. Physical examination revealed a granuloma of about $3 \times 4 \mathrm{~cm}^{2}$ size on left side of oral cavity mucosa. b. TEM revealed six oval-shaped phagocytosed yeast cells within distended histocytes. c. A typical septum (arrow pointed) in the organism, which was the characteristic structure of P. marrneffei.

\section{Author details}

Yuping Ran ${ }^{1 *}$, Wengying $\mathrm{Hu}^{1}$, Kaiwen Zhuang ${ }^{1}$, Mao $\mathrm{Lu}^{1}$, Jinghong Huang ${ }^{1}$, Fengni $\mathrm{Xu}^{1}$, Xiaoxi Xu ${ }^{1}$, Xia Hua ${ }^{1}$, Jebina Lama ${ }^{1}$, Xin $\operatorname{Ran}^{1}$, Yalin Dai ${ }^{2}$ and Song Lei ${ }^{3}$

*Address all correspondence to: ranyuping@vip.sina.com

1 Departments of Dermatovenereology, West China Hospital, Sichuan University, Chengdu, China

2 Departments of Medical Laboratory, West China Hospital, Sichuan University, Chengdu, China

3 Departments of Pathology, West China Hospital, Sichuan University, Chengdu, China 


\section{References}

[1] Liesegang TJ. Herpes simplex virus epidemiology and ocular importance. Cornea. 2001;20:1-13.

[2] Roizman B, Sears AE. Herpes simplex viruses and their replication. Fields Virol. 2001;2:2399-2459.

[3] Martinez V, Caumes E, Chosidow O. Treatment to prevent recurrent genital herpes. Curr Opin Infect Dis. 2008;21(1):42-48.

[4] Herpes zoster. Available at: http://en.wikipedia.org/wiki/herpes_zoster. Last updated March 9, 2015.

[5] Postlethwaite R. Molluscum contagiosum: A review. Arch Environ Health. 1970;21(3):432-452.

[6] Hanson D, Diven DG. Molluscum contagiosum. [electronic resource] Dermatol Online J. 2003;9(2).

[7] Rown J, Janniger CK, Schwartz RA, Silverberg NB. Childhood molluscum contagiosum. Int J Dermatol. 2006;45(2):93-99.

[8] Basta-Juzbašić A, Čeović R. Chancroid, lymphogranuloma venereum, granuloma inguinale, genital herpes simplex infection, and molluscum contagiosum. Clin Dermatol. 2014;32(2):290-298.

[9] Pardillo FE, Fajardo TT, Abalos RM, Scollard D, Gelber RH. Methods for the classification of leprosy for treatment purposes. Clin Infect Dis. 2007;44:1096.

[10] Leprosy. Available at: http://en.wikipedia.org/wiki/leprosy. Last updated April 9, 2015.

[11] Ellabib MS, Agaj M, Khalifa Z, Kavanagh K. Trichophyton violaceum is the dominant cause of tinea capitis in children in Tripoli, Libya: Results of a two year survey. Mycopathologia. 2001;153:145-147.

[12] Yu J, Li R, Bulmer G. Current topics of tinea capitis in China. Jpn J Med Mycol 2005;46:61-66.

[13] Zhuang K, Ran X, Lei S, et al. Scanning and transmission electron microscopic observation of the parasitic form of Trichophyton violaceum in the infected hair from tinea capitis. Scanning. 2014;36:465-470.

[14] Schwartz RA. Superficial fungal infections. Lancet. 2004;364:1173-1182.

[15] Shivaprakash MR, Singh G, Gupta P, et al. Extensive white piedra of the scalp caused by Trichosporon inkin: A case report and review of literature. Mycopathologia. 2011;172(6):481-486. 
[16] Kiken DA, Sekaran A, Antaya RJ, et al. White piedra in children. J Am Acda Dermatol. 2006;55:956-961.

[17] Khongkunthian P, Isaratanan W, Samaranayake LP, Gelderblom HR, Reichart PA. Case Report. Oro-facial manifestations of Penicillium marneffei infection in a Thai patient with AIDS. Mycoses. 2002;45(9-10):411-414.

[18] Hua X, Zhang R, Yang H, Lei S, Zhang Y, Ran Y. Primary oral Penicillium marneffei infection diagnosed by PCR-based molecular identification and transmission electron microscopic observation from formalin-fixed paraffin-embedded tissues. Med Mycol Case Rep. 2013;2:15-18. 